\title{
Gender variation in central serous chorioretinopathy
}

\author{
Daren Hanumunthadu ${ }^{1}$ - Elon H. C. Van Dijk ${ }^{2}$ Sankeert Gangakhedkar ${ }^{3}$ - Abhilash Goud ${ }^{3}$ • \\ Chui Ming Gemmy Cheung $\mathbb{1}^{4} \cdot$ Daniel Cherfan $^{5} \cdot$ Chintan Sarvaiya $^{6} \cdot$ Alay Banker $^{6} \cdot$ Catherine Meyerle $^{7}$. \\ Camiel J. Boon ${ }^{2} \cdot$ Rishi Singh ${ }^{5} \cdot$ Lihteh $\mathrm{Wu}^{8} \cdot$ Jay Chhablani $\mathbb{1}^{3}$
}

Received: 29 March 2018 / Revised: 6 May 2018 / Accepted: 27 May 2018 / Published online: 9 July 2018

(c) The Royal College of Ophthalmologists 2018

\begin{abstract}
Background Comparison of presentation and outcomes of central serous chorioretinopathy (CSC) between male and female subjects in different ethnic populations.

Methods Retrospective comparison between male and female subjects with CSC was completed. Demographic details, clinical presentations, imaging features and treatment outcomes were compared at baseline and at last follow-up.

Results This study included 155 male and 155 female subjects with a mean (CSD) age of $43.8 \pm 10.3$ and $57.0 \pm 12.1$ years, respectively, and a mean duration of follow-up of $8.49 \pm 12.6$ months. At presentation, there was no difference in visual acuity; however, visual acuity was significantly higher for female subjects at last follow-up $(p=0.02)$. Optical coherence tomography (OCT) analysis showed that subretinal deposits $(p<0.001)$, hyperreflective foci $(p=0.001)$, retinal pigment epithelial detachment $(p=0.01)$ and retinal pigment epithelium (RPE) irregularities $(p=0.03)$ were higher in male subjects at presentation. Angiographic analysis showed that diffuse leakage and RPE tracts were common in males $(p=0.01$ and $p=0.02$ ). No significant differences in choroidal dilatation or diffuse choroidal leakages were noted.

Conclusions Female subjects with CSC appear to have better outcomes, with less chances of diffuse RPE damage and other OCT features compared to males.
\end{abstract}

\section{Introduction}

Gender variation has been reported in a range of retinal diseases, but has a complex association dependent upon other demographic factors. For example, except in the

Jay Chhablan

jay.chhablani@gmail.com

1 Moorfields Eye Hospital NHS Foundation Trust, London EC1V 2PD, UK

2 Department of Ophthalmology, Leiden University Medical Center, Leiden, The Netherlands

3 Srimati Kanuri Santhamma Centre for Vitreo-Retinal Diseases, Hyderabad Eye Research Foundation, LV Prasad Eye Institute, Kallam Anji Reddy Campus, Hyderabad, India

4 Singapore National Eye Centre, 11 Third Hospital Avenue, Singapore 168751, Singapore

5 Clevelend Clinic Cole Eye Institute, 2022 E 105th Street, Cleveland, OH 44106, USA

6 Banker's Retina Clinic and Laser Centre, 5 Subhash Society, Ahmedabad 380009, India

7 Retina Division, Wilmer Eye Institute, Johns Hopkins University School of Medicine, Baltimore, MD, USA

8 Apdo 144-1225 Plaza Mayor, San José 1225, Costa Rica oldest age groups, gender has been reported to have similar prevalence in age-related macular degeneration (AMD) [1]. Interestingly, there has been a reported higher risk of neovascular AMD in women in a meta-analysis in European populations [2]. Conversely, no gender differences were shown in the prevalence of retinal vein occlusion in a recent cross-sectional observational study [3].

Central serous chorioretinopathy (CSC) is a chorioretinal disease characterized predominantly by serous detachment of the neurosensory retina at the macula. It has been associated with a number of risk factors including exogenous steroid use, obstructive sleep apnoea, pregnancy and male gender [4]. The preponderance for male gender as a risk factor for CSC has been evaluated in several studies [4-6]. The annual incidence of CSC, for example, in the Olmstead County, Minnesota population study was 9.9 for men and 1.7 for women per 100,000 individuals [7]. Similar findings were shown in studies in an Asian populations, although 
interestingly women were noted to present at an older age group [8].

Quantitative and qualitative analysis of optical coherence tomography (OCT)-derived images has revealed that there are a number of structural changes in CSC in addition to neurosensory detachment (NSD). Whilst subfoveal choroidal thickness (CT) has been shown to be higher in eyes with CSC, various morphological features have also been investigated including pigment epithelial detachment (PED), subretinal deposition and hyperreflective foci [9-13]. Variation in visual acuity outcomes has been suggested to be partly due to alterations in these structural features. Interestingly, there may be gender variation in particular morphological features of CSC, perhaps with associated change in visual acuity, prognosis or response to treatment [14].

Gender variation in OCT-derived features including macular thickness has been reported in normal subjects in a range of populations, although further evaluation in patients with retinal disease is required $[15,16]$. It is essential to describe structural changes associated with gender in order to help define prognosis and facilitate treatment decisions. The aim of this study was to investigate possible variations in visual acuity and retinal morphology associated with gender in adult subjects with CSC, including at presentation and follow-up.

\section{Subjects and methods}

This was a multicentric retrospective study that included evaluation of subjects diagnosed with CSC both at presentation and resolution of disease activity. The study was approved by the local ethics committee at all study sites and adhered to the tenets set forth in the Declaration of Helsinki.

\section{Study population}

Consecutive adult female subjects (aged $\geq 18$ years) diagnosed with naïve CSC with refractive error $\pm 3 \mathrm{D}$ and axial length $\leq 26 \mathrm{~mm}$ were identified from retina clinics from three participating sites (India, United States, Costa Rica), seen between January 2015 and June 2016. All data in this study were collected as part of routine ophthalmic examination of patients attending the clinic. Patients provided consent for their retrospective data to be included in this study. Exclusion criteria including history of exposure to steroids in the past 1 year, history of other active/previous chorioretinal disease, media opacity preventing adequate fundal view, previous ocular surgery (other than cataract surgery) and other significant ocular comorbidity. Age-matched males with the same inclusion and exclusion criteria were also selected.

\section{Imaging protocol}

Best-corrected visual acuity (BCVA) was determined and medical comorbidity was recorded from patient's notes. All subjects underwent a comprehensive ophthalmic examination. All subjects were adequately dilated with $2.5 \%$ phenylephrine and $1 \%$ tropicamide prior to imaging. Each patient underwent SD-OCT (Cirrus, Carl Zeiss Meditec, Dublin, CA, USA or Spectralis, Heidelberg Engineering, Heidelberg, Germany) volume scan of the macula, fundus fluorescein angiography (FFA) and indocyanine green angiography (ICGA) (as per physician's discretion). Visual acuity assessment and imaging was repeated at follow-up visit after resolution of disease activity.

\section{SD-OCT quantitative analyses}

All SD-OCT macula images were exported in jpeg format and analysed manually using calipers on the ImageJ software (a publicly available image processing program developed by Wayne Rashand, National Institute of Health, Bethesda, MD, USA, available at: http://rsb.info.nih.gov/ij/ index.html) by a single observer (AG). In addition to central macular thickness (CMT), subfoveal CT was determined as the distance between the underside of the retinal pigment epithelium (RPE)-Bruch's membrane complex and choroidal-scleral interface. The height of the NSD was measured at the fovea as the perpendicular distance between the RPE and neurosensory retina. Intra-observer repeatability of 0.98-0.99 was noted for all measurements.

\section{Qualitative analyses of retinal features of CSC}

SD-OCT images were evaluated by an independent observer (JC) for reported qualitative features associated with CSC (Fig. 1) including subretinal deposits, RPE irregularities, PED, double layer sign, outer retinal layer disruption, hyperreflective foci, intraretinal fluid and choroidal neovascular membrane (CNV). All line scans were individually assessed and noted for the presence of the individual retinal feature.

FFA images were scrutinized for the presence of sites of single/multiple leakage, diffuse leakage, tracts and CNV. ICG images were assessed for choroidal vessel dilatation, diffuse and point leakage.

\section{Statistical analyses}

Differences between male and female visual acuity, quantitative and qualitative features of imaging were compared with Mann-Whitney test and Fisher's exact test. All statistical analyses were completed in GraphPad Prism Version 5. 


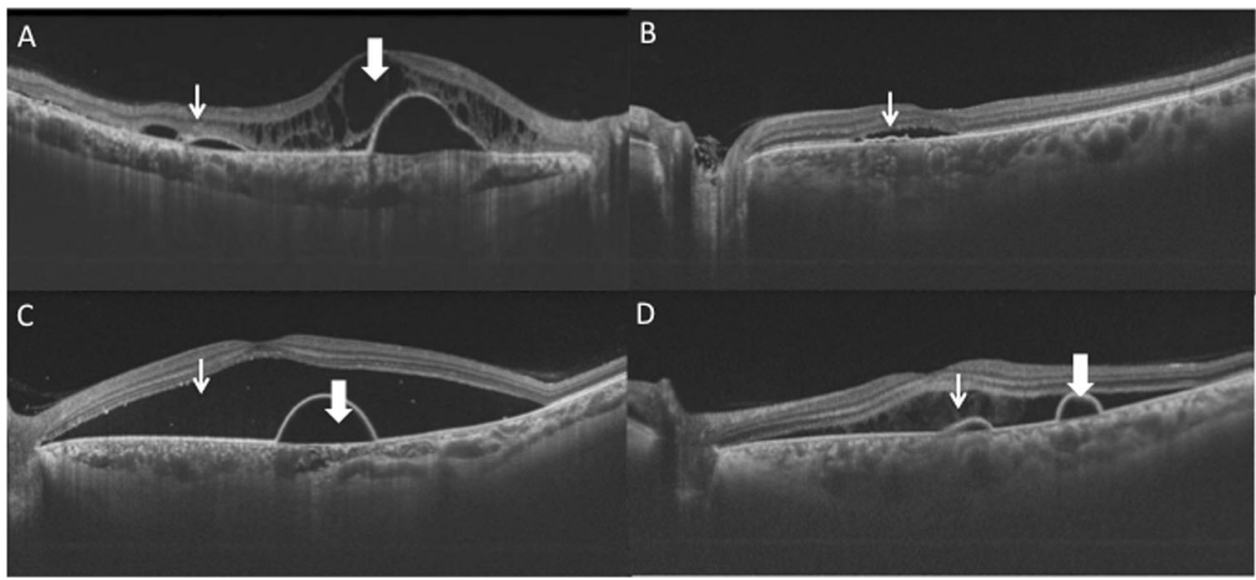

Fig. 1 Qualitative spectral-domain optical coherence tomography features in central serous chorioretinopathy evaluated in subjects. SDOCT images were manually evaluated by an independent observer for reported qualitative features associated with CSC (Fig. 1) including chronic cystoid changes (thick arrow) and hyperreflective deposits

\section{Results}

\section{Subject characteristics}

This study included 155 male and 155 female subjects from a range of different ethnicities and 155 left and 155 right eyes. Mean follow-up of male and female subjects was $45.9 \pm 11.6$ and $57.0 \pm 12.1$ weeks. The ethnic distribution of the subjects (and corresponding proportion of gender) included Caucasian $\quad$ (male $=40,25.8 \%$ female $=67$, $43.2 \%)$, Hispanic $($ male $=28,18.1 \%$ female $=25,16.1 \%)$ and Asian $(\mathrm{male}=84,54.1 \%$ female $=51,32.9 \%)$. The visual acuity of male and female subjects at presentation was not significantly different at presentation (male: 0.42, female $0.38 ; p=0.62$ ), but female subjects appeared to have significantly better visual acuity at follow-up (male 0.24 , female $0.14 ; p=0.04$ ). The mean duration of the disease in male $(7.3 \pm 4.1)$ subjects was significantly less than female $(18.7 \pm 6.9)$ subjects $(p=0.02)$. All subject characteristics are summarized in Table 1.

\section{SD-OCT quantitative parameters}

SD-OCT-derived CMT measurements appeared to be greater in male $(419.8 \pm 173.2 \mu \mathrm{m})$ than female subjects $(367.8 \pm 158.4 \mu \mathrm{m})$ at presentation $(p=0.03)$. At follow-up, there was no significant difference between CMT measurements between male and female subjects $(p=0.12)$. Subfoveal CT in male subjects was significantly larger than female subjects at presentation $(p=0.01)$ but not at followup $(p=0.41)$. There was no significant difference between the NSD height either at presentation or follow-up between (thin arrow) (a), double layer sign (b), pigment epithelial detachment (thick arrow) and neurosensory detachment (thin arrow) (c), subretinal fibrin (thin arrow) and retinal pigment epithelial detachment (thick arrow) (d)

Table 1 Subject demographics

\begin{tabular}{lll}
\hline & Male & Female \\
\hline $\begin{array}{l}\text { Number of subjects } \\
\text { Laterality }(n, \%)\end{array}$ & 155 & 155 \\
Left & $80(51.6)$ & $74(47.7)$ \\
Right & $75(48.4)$ & $81(52.3)$ \\
Age at presentation & & \\
Mean \pm SD (years) & $43.8 \pm 10.3$ & $57.0 \pm 12.1$ \\
Ethnicity (n, \%) & & \\
Caucasian & $40(25.8)$ & $67(43.2)$ \\
Asian & $84(54.1)$ & $51(32.9)$ \\
Hispanic & $28(18.1)$ & $25(16.1)$ \\
Afro-Carribean & $3(1.9)$ & $7(4.5)$ \\
Chinese & 0 & $5(3.2)$ \\
Duration of disease (mean \pm SD, weeks) & $7.3 \pm 4.1$ & $18.7 \pm 6.9$ \\
\hline
\end{tabular}

Number of subjects, laterality, ethnicity, follow-up of subjects with central serous chorioretinopathy included in the study

male and female subjects ( $p=0.08$ and 0.37 , respectively). SD-OCT quantitative parameters are described in Table 2.

\section{SD-OCT and angiography qualitative features}

\section{SD-OCT features}

Analysis of SD-OCT images showed that the prevalence of subretinal deposits $(p<0.001)$ and $\operatorname{PED}(p=0.01)$ were greater in male than female subjects at presentation but no difference was noted at follow-up (Fig. 2). The prevalence of RPE irregularities and hyperreflective foci were higher in male subjects at presentation $(p=0.03$ and $p=0.001$, 
respectively) and at follow-up ( $p=0.02$ and $p=0.01$ respectively). There was no significant difference in the presence of double layer sign, outer retinal layer disruption, intraretinal foci and CNV between male and female subjects at presentation or follow-up.

\section{FFA features}

FFA analysis (Fig. 3) showed that diffuse RPE leakage and RPE tracts was more prevalent in male subjects at presentation ( $p=0.01$ and 0.03 , respectively) and at follow-up ( $p=0.03$ and $p=0.001$, respectively). There was no significant difference in $\mathrm{CNV}$ presence or in the number of

Table 2 Comparison of quantitative spectral-domain optical coherence tomography parameters between male and female subjects

\begin{tabular}{|c|c|c|c|}
\hline & Male & Female & $P$ value \\
\hline \multicolumn{4}{|c|}{ Central macular thickness $(\mu m)$} \\
\hline Presentation & $419.8 \pm 173.2$ & $367.8 \pm 158.4$ & 0.03 \\
\hline Follow-up & $298.0 \pm 131.2$ & $306.1 \pm 111.4$ & 0.12 \\
\hline \multicolumn{4}{|c|}{ Subfoveal choroidal thickness $(\mu \mathrm{m})$} \\
\hline Presentation & $339.5 \pm 141.1$ & $298.3 \pm 136.7$ & 0.01 \\
\hline Follow-up & $319.7 \pm 123.6$ & $299.5 \pm 111.5$ & 0.41 \\
\hline \multicolumn{4}{|c|}{ Presence of neurosensory detachment $(n, \%)$} \\
\hline Follow-up & $50(32.3)$ & $31(20.0)$ & 0.74 \\
\hline \multicolumn{4}{|c|}{ Neurosensory detachment $(\mu \mathrm{m})$} \\
\hline Presentation & $255.7 \pm 195.7$ & $197.5 \pm 152.1$ & 0.08 \\
\hline Follow-up & $101 \pm 140.4$ & $116.1 \pm 122.2$ & 0.37 \\
\hline
\end{tabular}

A

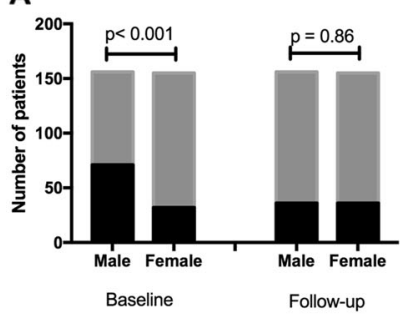

E

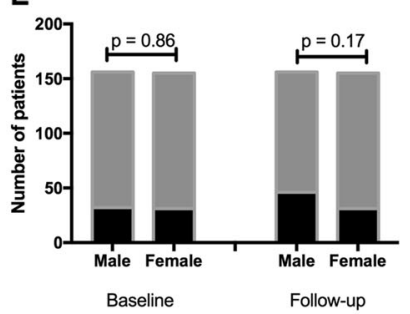

B

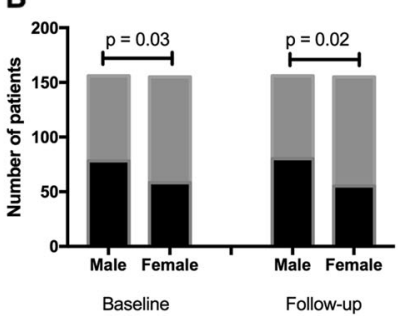

$\mathbf{F}$

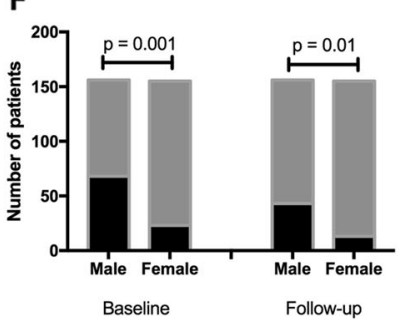

Fig. 2 Gender variation in optical coherence tomography-derived qualitative features of central serous chorioretinopathy at baseline and follow-up. Presence (black) and absence (grey) of spectral-domain optical coherence tomography features of central serous chorioretinopathy showing difference between gender at baseline and follow- single/multiple areas of leakage between male and female subjects during the study.

\section{ICGA features}

The prevalence of leakage on ICG (Fig. 3) was higher in male subjects at presentation $(p=0.01)$ and at follow-up $(p=0.03)$. There was no significant difference in the prevalence of choroidal dilatation and diffuse leakage between male and female subjects throughout the study (all $p>0.05)$.

\section{Analysis of ethnic variation}

There was no significant difference between BCVA measurements at presentation between the three most prevalent ethnicities $($ Indian $=0.14$, Caucasian $=0.14$ and Asian $=$ 0.18 ), $p>0.05$ (analysis of variance (ANOVA)). There was also no significant difference between CMT and height of NSD between the gender. Subfoveal CT appeared to vary between the genders at presentation (Caucasian $=330.4 \pm$ $112.3 \mu \mathrm{m} ; \quad$ Asian $=223.2 \pm 106.1 \mu \mathrm{m}, \quad$ Afro-Carribean $=$ $301.2 \pm 118.1 \mu \mathrm{m} ;$ Hispanic $=296.5 \pm 117.3 \mu \mathrm{m}, p=0.03$, ANOVA). Analysis of OCT features showed that there was no significant difference in the OCT or angiographic features included in this study at baseline.

\section{Discussion}

We report the variation in chorioretinal structure and associated visual acuity between male and female subjects with
C

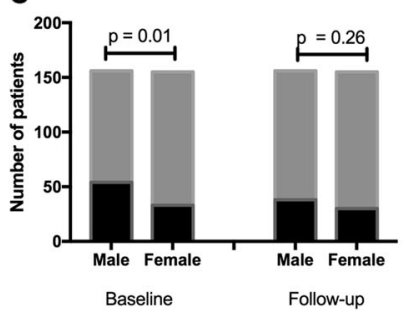

G

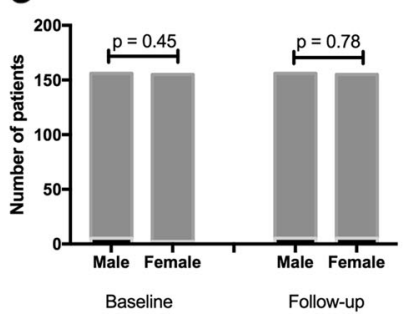

D

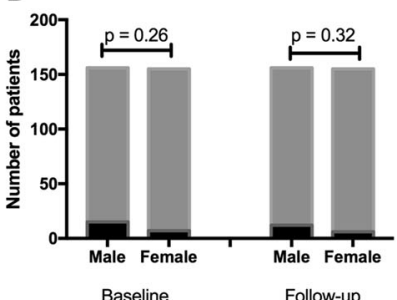

H

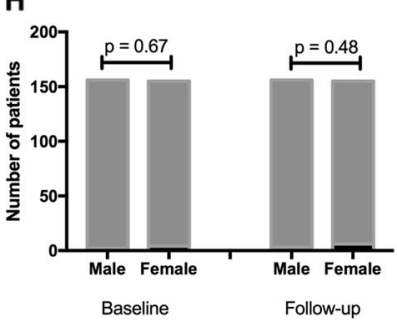

up including (a) subretinal deposits, b retinal pigment epithelium irregularities, c pigment epithelial detachment, d double layer sign, e outer retinal layer disruption, $\mathbf{f}$ hyperreflective foci, $\mathbf{g}$ intraretinal fluid and (h) choroidal neovascular membrane (Fisher's exact test) 
A

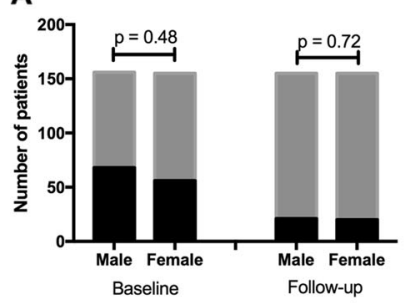

E

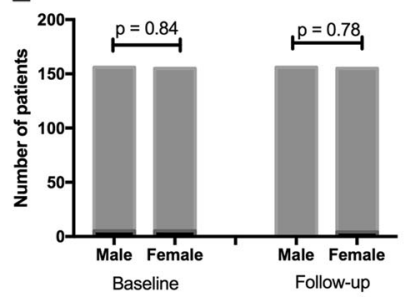

B

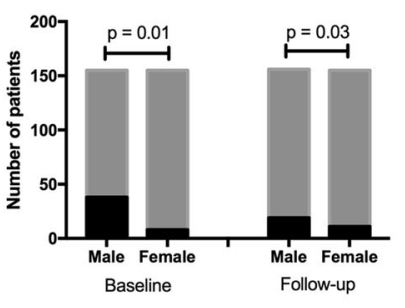

F

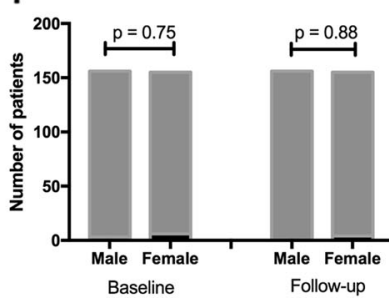

Fig. 3 Gender variation in angiographic qualitative features of central serous chorioretinopathy at baseline and follow-up. Presence (black) and absence (grey) of features of central serous chorioretinopathy at baseline and follow-up: a single leaks, b diffuse leakage, $\mathbf{c}$ RPE tracts,

CSC in a range of different ethnic populations. There was no significant difference in visual acuity at presentation, but female patients had significantly better visual acuity at follow-up. This better visual outcome in female patients appears to be associated with a reduction in alterations in chorioretinal structure compared with male subjects.

Quantitiative analysis of SD-OCT images showed that although there was no significant difference in height of NSD, male subjects appeared to have larger retinal thickness compared with female subjects at baseline, although this difference was not statistically significant at the follow-up visit. This could suggest that the baseline retinal thickness is more important for final visual acuity outcomes as female patients also appeared to have better visual acuity at follow-up. CT was larger in male patients compared to female subjects at presentation (but not follow-up), suggesting that choroidal thickening may be a factor in visual function. CT alteration is typical of patients with CSC; previous studies suggest that $\mathrm{CT}$ is greater in eyes with CSC and fellow eyes compared with age-matched controls [9, 17].

Qualitative analysis of SD-OCT images showed that there were less hyperreflective foci in female subjects and reduced RPE alteration (with fewer subretinal deposits, PED, RPE irregularities). Similarly analysis of FA and ICGA showed that there was more RPE disruption in male patients with diffuse RPE leakage, RPE tracts and point leakage. These alterations in retinal morphology may also be a factor in the poorer visual acuity outcomes in male subjects. Indeed, subretinal deposits themselves in CSC have been correlated with poorer visual function [12]. Interestingly, female patients appeared to show a reduction in subretinal deposits at follow-up compared with the male

C

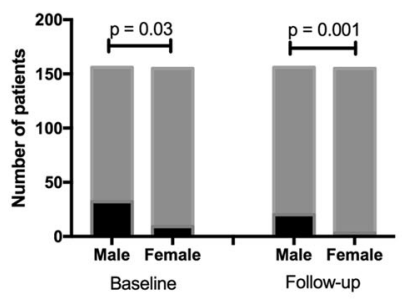

D

G

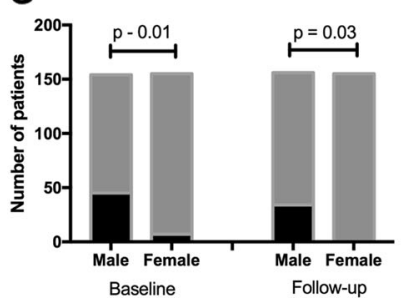

d choroidal neovascular membrane at baseline, e choroidal vessel dilatation, $\mathbf{f}$ choroidal leakage and $\mathbf{g}$ choroidal point leakage (Fisher's exact test)

subjects, suggesting perhaps that these factors may be of use in the assessment of disease chronicity. Alteration in RPE structure on SD-OCT and more leakage (indicating RPE disruption) on angiography were both demonstrated at both baseline and follow-up in male patients (compared with female subjects), suggesting that there is persistent alteration in chorioretinal structure.

Gender differences have been evaluated in a range of retinal diseases and a similar complex relationship has been demonstrated. Indeed, epidemiological studies have suggested that AMD appears more prevalent in female individuals in many studies, although similar studies in Asia have found a higher prevalence in male patients [2, 18, 19]. Male gender has been suggested, however, as a possible protective factor in AMD [20]. Structural variations do not necessarily reflect these visual acuity trends; it has been suggested that features of early AMD appear more consistently in patients with male patients [21]. Diabetic retinopathy has been shown to be more prevalent in men in various studies, although this may also be a reflection of ethnic variation in glycaemic control, blood pressure and treatment patterns of diabetes [22]. It is clear that further study of structural associations with gender in a range of retinal diseases needs further evaluation.

Our analysis of gender variation showed that visual acuity at presentation was not different across the most prevalent ethnicities in this study (Asian, Caucasian and Hispanic). There was no significant difference between the quantitative and qualitative features at baseline except for $\mathrm{CT}$, suggesting that ethnic variation was not responsible for the differences noted in this study.

Limitations of our study include its retrospective design. However, we were able to include a significant number of 
patients with a range of imaging modalities. One of the unique strengths of this study is that we incorporated subjects from a range of different ethnic populations who were seen both at presentation and follow-up. There were relatively few number of Afro-Caribbean participants in our study; it would be useful to investigate our findings further in Afro-Caribbean patients with CSC. We excluded subjects with steroid exposure to assess the characteristics of primary CSCR.

To the best of our knowledge, this is the first study to describe visual outcome and the structural variation between genders using SD-OCT, FFA and ICGA both at presentation and follow-up. In summary, we report that female subjects appeared to have better visual acuity outcomes at follow-up with associated reduced alteration in retinal morphology including RPE disruption. It would be interesting to investigate how the structural variations reported in our paper could be affected by treatment. This can be used to help define treatment decisions particularly by assessment of the chronicity of disease.

\section{Summary}

\section{What was known before}

- Qualitative analysis of subjects with CSC indicates significant variation in chorioretinal structure.

- Gender variation has been reported in both OCT-derived structural parameters and visual outcomes in range of chorioretinal diseases.

\section{What this study adds}

- Female subjects appear to have better visual outcomes.

- Female subjects appear to have reduced OCT-derived structural changes particularly retinal pigment epithelium damage.

Author contributions All authors were involved in the design of the project (or the acquisition of the data), drafting of the article and final approval of the version to be published.

\section{Compliance with ethical standards}

Conflict of interest The authors declare that they have no conflict of interest.

\section{References}

1. Colijn JM, Buitendijk GHS, Prokofyeva E, Alves D, Cachulo ML, Khawaja AP, et al. Prevalence of age-related macular degeneration in Europe: the past and the future. Ophthalmology. 2017;124: 1753-63.
2. Rudnicka AR, Jarrar Z, Wormald R, Cook DG, Fletcher A, Owen CG. Age and gender variations in age-related macular degeneration prevalence in populations of European ancestry: a metaanalysis. Ophthalmology. 2012;119:571-80.

3. Shin JY, Kwon KY, Byeon SH. Association between choroidal thickness and the response to intravitreal ranibizumab injection in age-related macular degeneration. Acta Ophthalmol. 2015;93:524-32.

4. Chatziralli I, Kabanarou SA, Parikakis E, Chatzirallis A, Xirou T, Mitropoulos P. Risk factors for central serous chorioretinopathy: multivariate approach in a case-control study. Curr Eye Res. 2017;42:1069-73.

5. Spaide RF, Campeas L, Haas A, Yannuzzi LA, Fisher YL, Guyer $\mathrm{DR}$, et al. Central serous chorioretinopathy in younger and older adults. Ophthalmology. 1996;103:2070-2079. discussion 2079-80

6. Matet A, Dariuch A, Zola M, Behar Cohen F. Risk factors for recurrences of central serous chorioretinopathy Retina $2018 \mathrm{Jul}$; 38(7):1403-1414

7. Kitzmann AS, Pulido JS, Diehl NN, Hodge DO, Burke JP. The incidence of central serous chorioretinopathy in Olmsted County, Minnesota, 1980-2002. Ophthalmology. 2008;115:169-73.

8. Tsai DC, Chen SJ, Huang CC, Chou P, Chung CM, Huang PH, et al. Epidemiology of idiopathic central serous chorioretinopathy in Taiwan, 2001-2006: a population-based study. PLoS ONE. 2013;8:e66858.

9. Kuroda S, Ikuno Y, Yasuno Y, Nakai K, Usui S, Sawa M, et al. Choroidal thickness in central serous chorioretinopathy. Retina. 2013;33:302-8.

10. Yang L, Jonas JB, Wei W. Optical coherence tomography-assisted enhanced depth imaging of central serous chorioretinopathy. Invest Ophthalmol Vis Sci. 2013;54:4659-65.

11. Mitarai K, Gomi F, Tano Y. Three-dimensional optical coherence tomographic findings in central serous chorioretinopathy. Graefe's Arch Clin Exp Ophthalmol. 2006;244:1415-20.

12. Landa G, Barnett JA, Garcia PM, Tai KW, Rosen RB. Quantitative and qualitative spectral domain optical coherence tomography analysis of subretinal deposits in patients with acute central serous retinopathy. Ophthalmologica. $2013 ; 230: 62-68$.

13. Ahlers C, Geitzenauer W, Stock G, Golbaz I, Schmidt-Erfurth U, Prunte C. Alterations of intraretinal layers in acute central serous chorioretinopathy. Acta Ophthalmol. 2009;87:511-6.

14. Kunavisarut P, Pathanapitoon K, van Schooneveld M, Rothova A. Chronic central serous chorioretinopathy associated with serous retinal detachment in a series of Asian patients. Ocul Immunol Inflamm. 2009;17:269-77.

15. Patel PJ, Foster PJ, Grossi CM, Keane PA, Ko F, Lotery A, et al. Spectral-domain optical coherence tomography imaging in 67321 adults: associations with macular thickness in the UK Biobank Study. Ophthalmology. 2016;123:829-40.

16. von Hanno T, Lade AC, Mathiesen EB, Peto T, Njolstad I, Bertelsen G. Macular thickness in healthy eyes of adults $(N=4508)$ and relation to sex, age and refraction: the Tromso Eye Study (2007-2008). Acta Ophthalmol. 2017;95:262-9.

17. Maruko I, Iida T, Sugano Y, Ojima A, Sekiryu T. Subfoveal choroidal thickness in fellow eyes of patients with central serous chorioretinopathy. Retina. 2011;31:1603-08.

18. Smith W, Mitchell P, Wang JJ. Gender, oestrogen, hormone replacement and age-related macular degeneration: results from the Blue Mountains Eye Study. Aust NZ J Ophthalmol. 1997;25 Suppl 1:S13-15.

19. You QS, Xu L, Yang H, Li YB, Wang S, Wang JD, et al. Fiveyear incidence of age-related macular degeneration: the Beijing Eye Study. Ophthalmology. 2012;119:2519-25. 
20. Srinivasan S, Swaminathan G, Kulothungan V, Raman R, Sharma T. Prevalence and the risk factors for visual impairment in agerelated macular degeneration. Eye (Lond). 2017;31:846-55.

21. Brandl C, Breinlich V, Stark KJ, Enzinger S, Assenmacher M, Olden M, et al. Features of age-related macular degeneration in the general adults and their dependency on age, sex, and smoking: results from the German KORA Study. PLoS ONE. 2016;11: e0167181.

22. Mehlsen J, Erlandsen M, Poulsen PL, Bek T. Identification of independent risk factors for the development of diabetic retinopathy requiring treatment. Acta Ophthalmol. 2011;89:515-21. 